\title{
Study on the influence of technological parameters on 3D printing with sla technology
}

\author{
Andrei Komjaty $^{1 *}$, Elena Stela Wisznovszky (Muncut) ${ }^{1}$, and Lavinia Ioana Culda ${ }^{1}$ \\ ${ }^{1}$ University “Aurel Vlaicu” of Arad, B-dul Revoluţiei Nr. 77, 310130, România.
}

\begin{abstract}
The influence of the technological parameters of the printing through the SLA (Stereolithography) technology is presented. This printing technology is based on the use of a photosensitive resin, which polymerizes in contact with UV rays with a wavelength of $405 \mathrm{~nm}$. An Anycubic Photon Mono printer is used, on which parts are printed, in which the dimensional accuracy and the condition of the resulting surfaces will be analyzed. It will study the influence of the polymerization time of the resin $(5 \mathrm{~s}-10 \mathrm{~s})$, of the advance step between the successively deposited layers $(0.05 \mathrm{~mm}-0.2 \mathrm{~mm})$, as well as the influence of the placement positioning of the reference mark for printing. A Black basic type, monomer with photo-initiator photosensitive resin will be used made by Anycubic,. A Sony alpha 37 DSLR camera with Sony SAL-100M28 $100 \mathrm{~mm}$ F / 2.8 AF Macro lens will be used to capture images for the resulting surfaces.
\end{abstract}

\section{Introduction}

The general concept and the procedure to be used in 3D-printing was first described by Raymond F. Jones in his short story, "Tools of the Trade," published in the November 1950 issue of Astounding Science Fiction magazine. He referred to it as a "molecular spray" in that story $[1,2,3]$. Stereolithography or "SLA" printing is an early and widely used 3D printing technology. In the early 1980s, Japanese researcher Hideo Kodama first invented the modern layered approach to stereolithography by using ultraviolet light to cure photosensitive polymers [4].

Stereolithography is a rapid prototyping process that is based on focusing a beam of UV light, generated by a high-intensity LED with a wavelength of $405 \mathrm{~nm}$ on a layer of photosensitive resin, located in a tank with a transparent bottom. In this way the piece is printed in reverse "bottom up" position. The piece is created on a platform that is immersed in the resin, until it reaches the bottom of the tray, and will rise with the thickness of the layer "layer". We can distinguish "base layers", which are kept at a longer exposure time to the UV beam, to create a more solid base, which does not come off the aluminum plate at the printer. Once the printed layer is polymerized, it sticks to the bottom of the tray, so that for each layer made, it is necessary to detach the piece from it. This process is repeated every layer built, until the piece is finished. Due to these release forces, you must choose

* Corresponding author: komjatya@gmail.com 
the correct printing position, so that you do not have much larger surfaces to detach from the bottom of the tray than the base surface. If, however, there are such surfaces, it is recommended to use support to increase the adhesion forces of the mobile platform.

An Anycubic Photon Mono printer will be used. Anycubic company was founded in 2015, with a number of 300 employees, being located in Shenzhen, China [5,6]. The main features of the printer are: the working space of the printer is $130 \times 80 \times 165 \mathrm{~mm}$, the resolution in the XOY plane is $0.051 \mathrm{~mm}$, the step between layers (layer resolution), on the $\mathrm{OZ}$ axis, is $0.01-0.15 \mathrm{~mm}$., Connectivity - USB stick.

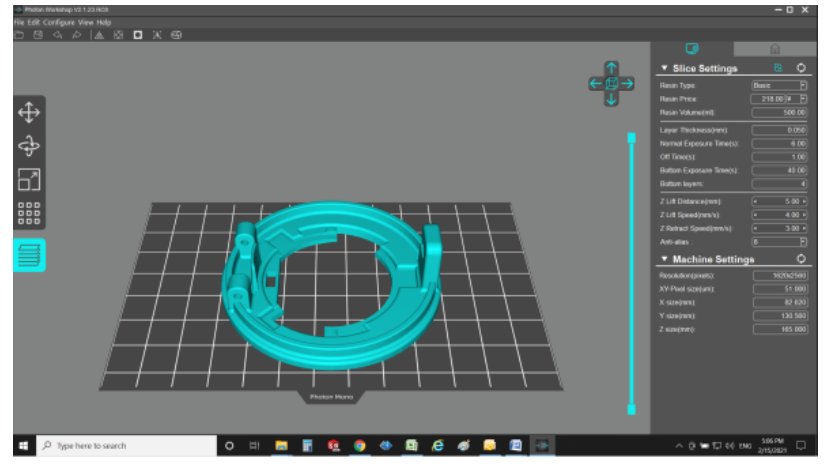

Fig. 1. Work piece position on the printers table.

The model we want to print must be made in a CAD software, or the desired object will be scanned with a $3 \mathrm{~d}$ scanner. The model thus made will be saved in a file in stereolithography file format (STL). The STL file will be opened with the Photon Workshop 64 program, in which the print position (figure 1), and the location of the support will be established, if necessary the definition of the technological parameters will be established too (figure 2). This software (slicer) will generate a * pwmo file. This file will be copied to a USB stick, which will be inserted into the Anycubic Photon Mono printer.

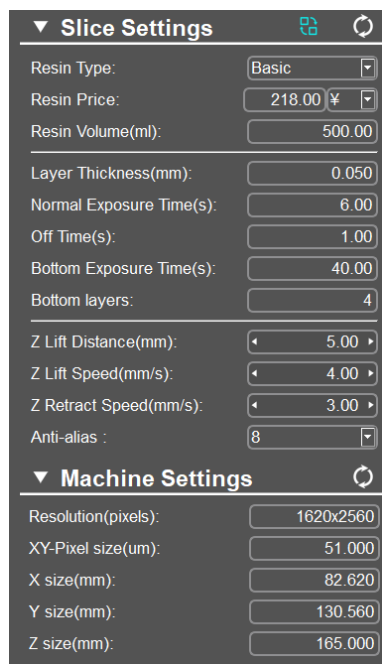

Fig. 2. Technological parameters. 


\section{Carrying out the tests}

The study was performed on different printed objects, varying the technological parameters one by one. The samples were measured with a micrometer / caliper, with an accuracy of $0.01 \mathrm{~mm}[7,8]$. Photographs were taken of the samples maintaining a constant focusing distance, using the same light source, located in the same position, keeping the shutter speed, aperture and ISO sensitivity at constant values, thus guaranteeing images with the same magnification and brightness. Black Basic resin was used for all tests. The recommended printing parameters are show in table 1.

Table 1. Recommended printing parameters

\begin{tabular}{|l|l|l|}
\hline \multicolumn{1}{|c|}{ Range } & \multicolumn{1}{c|}{ Minimum value } & \multicolumn{1}{c|}{ Maximum value } \\
\hline Parameters & 20 & 60 \\
\hline Bottom layer exposure time [s] & 5 & 15 \\
\hline Normal exposure time [s] & 0.02 & 0.15 \\
\hline Layer thickness [mm] &
\end{tabular}

In order to study the influence of the orientation position, all the technological parameters were maintained at the same value, varying only the position of the sample, achieving the two marks in a single print, one in horizontal position and one in vertical position. For the piece positioned in the vertical position, (the blue one), a support was used for the areas where this is necessary, the support being represented in brown. The orientation of the parts and the support for support can be seen in figure 3 .

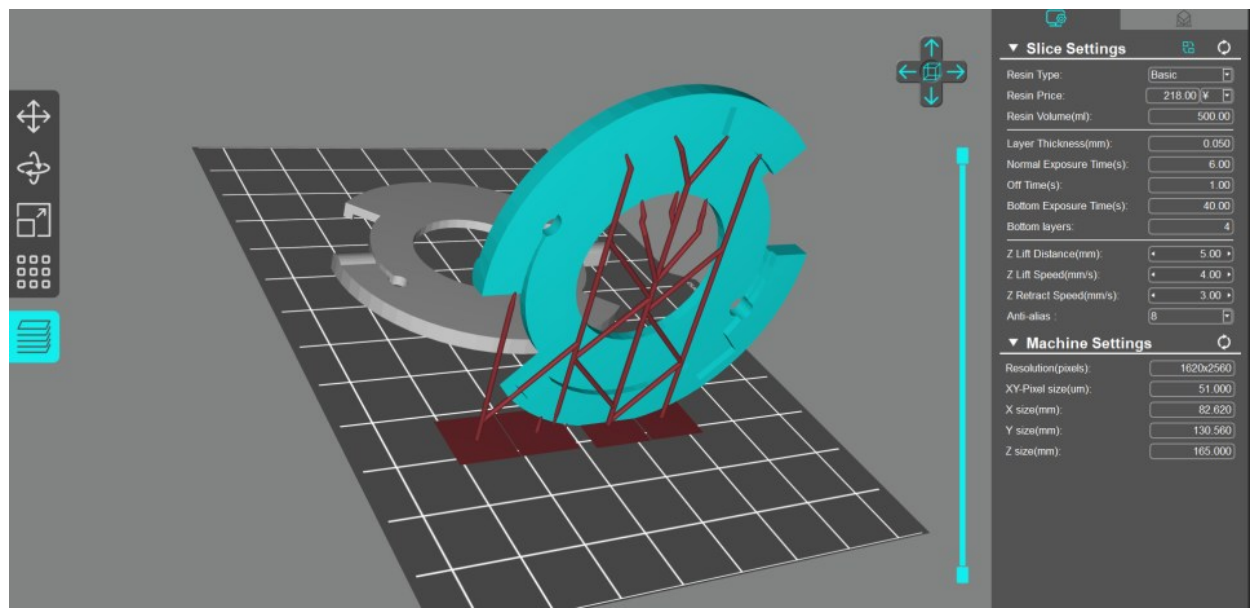

Fig. 3. Different work piece orientation.

\section{The obtained results}

For the reference in figure 4, the sizes for which the study was made were established, these being circled: 


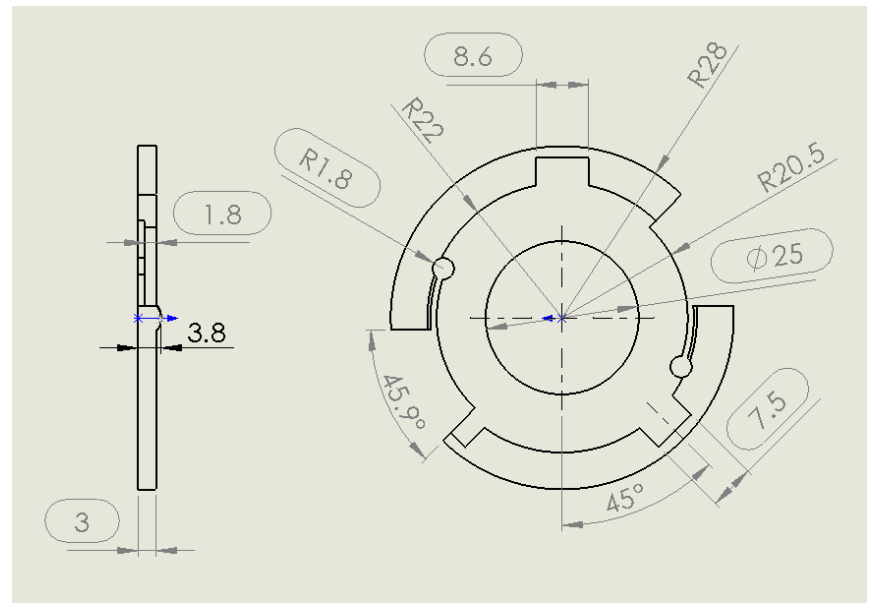

Fig. 4. Dimensions to be studied.

The dimensions were measured in 5 different points, the resulting dimension, which will be entered in the tables being an arithmetic mean of the measurements.

The measured values of the dimensions, depending on the position of the part in the type of printing, are listed in table 2:

Table 2. Dimensions by orientation

\begin{tabular}{|l|l|l|l|l|l|l|}
\hline \multicolumn{1}{|c|}{ Dimension } & 1.8 & 3 & R1.8 & 7.5 & 8.6 & 25 \\
\hline Orietation & & & & & & \\
\hline Horizotal & 1.86 & 2.95 & 1.67 & 7.4 & 8.54 & $\mathbf{2 4 . 8 3}$ \\
\hline Vertical & 1.85 & $\mathbf{3 . 0 8}$ & $\mathbf{1 . 6 8}$ & $\mathbf{7 . 4 6}$ & $\mathbf{8 . 4 5}$ & $\mathbf{2 4 . 9 6}$ \\
\hline
\end{tabular}

Next, we proceeded to the calculation of the execution tolerances depending on the orientation position of the work piece. This was calculated with the relation:

$$
T=\left|X_{\text {teor. }}-X_{\text {real }}\right|
$$

In the table 3 we have passed the values of the execution tolerances for the different cases of positions:

Table 3. Tolerances by orientation

\begin{tabular}{|l|l|l|l|l|l|l|}
\hline \multicolumn{1}{|c|}{ Dimension } & 1.8 & 3 & R1.8 & 7.5 & 8.6 & 25 \\
\hline Orietation & & & & & & \\
\hline Horizotal & 0.06 & 0.05 & 0.13 & 0.1 & 0.06 & 0.17 \\
\hline Vertical & $\mathbf{0 . 0 5}$ & $\mathbf{0 . 0 8}$ & $\mathbf{0 . 1 2}$ & $\mathbf{0 . 0 4}$ & $\mathbf{0 . 0 5}$ & $\mathbf{0 . 0 4}$ \\
\hline
\end{tabular}

Figure 5 shows a photograph of the parts printed in different positions:
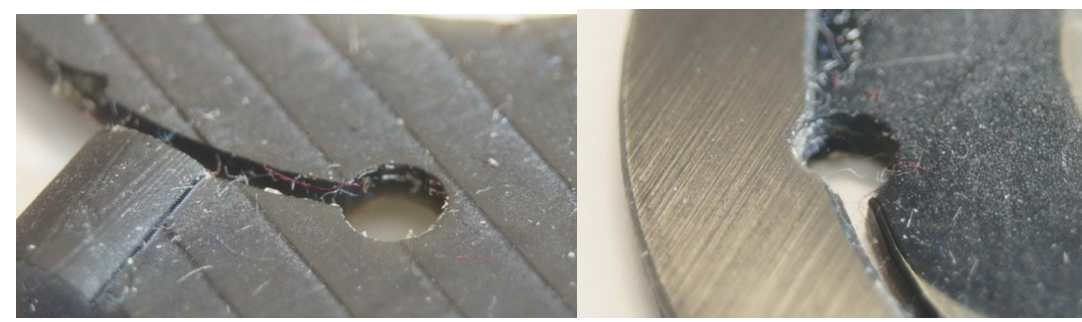

Fig. 5. Printed part in vertical (left) and horizontal (right) position 
In the case of the piece printed in vertical plane, it is necessary to remove the support layer (fig. 6) by mechanical processing operations by cutting.

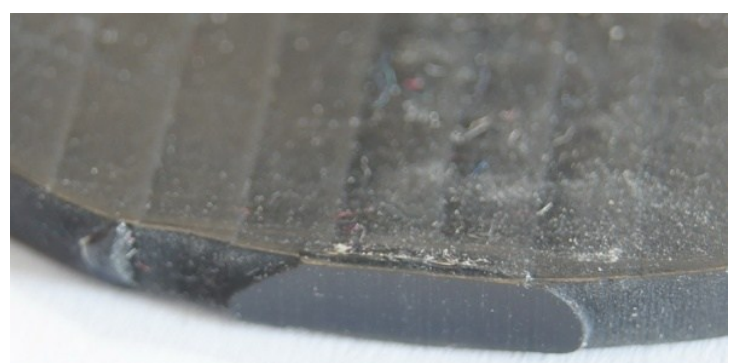

Fig. 6. Support for the piece from the vertical position

Regarding the influence of the feed rate on the OZ axis, the distance between the layers, can be observed from figure 7, with the increase of the advance will result a lower quality surface, higher roughness. In figure $7 \mathrm{left}$, we used a distance between layers of $0.15 \mathrm{~mm}$, obtaining a roughness of $\mathrm{Ra} 6.3 \mu \mathrm{m}$, and in the right case we used a distance of $0.05 \mathrm{~mm}$ between layers, obtaining a roughness of Ra1.6 $\mu \mathrm{m}$.
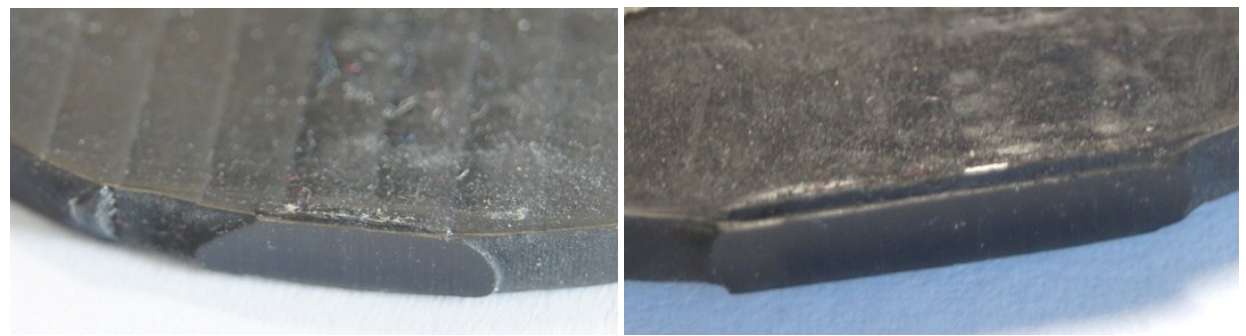

Fig. 7. The influence of the feed rate on $\mathrm{OZ}$ axis

Regarding the influence of exposure time I don't see any dependence of dimensions and the exposure time. If the bottom exposure time is not enough long the print can drop from the plate and the printing process must be ended. We can observed the differences of tensile strength and hardness are slightly lower than usual if the UV curing time [9] is too short and the curing temperature is too low. The results of hardness test [10] in different curing parameters are shown in table 4.

Table 4. Dependence between curing parameters

\begin{tabular}{|c|c|c|}
\hline \multicolumn{2}{|c|}{ Curing parameters } & \multirow{2}{*}{ Hardness [shore D] } \\
\hline Time [min] & Temperature $\left[{ }^{0} \mathbf{C}\right]$ & 60 \\
\hline 5 & 30 & 65 \\
\hline 5 & 100 & 76 \\
\hline 15 & 30 & 80 \\
\hline 15 & 100 & 77 \\
\hline 30 & 30 & 80 \\
\hline 30 & 100 & \\
\hline
\end{tabular}




\section{Conclusions}

In general, it can be said that the external dimensions achieved are larger than those of the model, and the internal dimensions made are smaller than the dimensions of the model. An explanation of this phenomenon would be that the polymerization of the resin is achieved not only with direct light from the LED source of the printer but also from the reflection of UV rays through the already polymerized photosensitive resin.

It can also be seen that the printing of the parts in the vertical plane is more precise than the horizontal position, this can also be explained by the dendritic polymerization of the photosensitive resin.

Regarding the appearance of the surfaces, it can be stated that printing the pieces in the vertical plane (figure 5 on the left) we will obtain a higher roughness than in the case of the surfaces printed in the horizontal plane. The lowest roughness is obtained if the surface is flat and it detaches by peeling off the bottom of the tray (figure 5 on the right). In the case of vertical surfaces, it can be stated that with the increase of the step on the $Z$ axis ( $Z$ lift) a degradation of the surface quality is obtained.

Regarding the hardness of surface, we must use the post printing cure with UV light and a higher temperature is better.

\section{References}

1. https://en.wikipedia.org/wiki/3D_printing

2. D. O. Glăvan, T. Babanatsas, R. M. Babanatis Merce and A. Glăvan - Comparative study of tool machinery sliding systems; comparison between plane and cylindrical basic shapes, IOP Conf. Series: Materials Science and Engineering 294 (2017)

3. L. I. Culda, E. S. Muncut, and A. Komjaty - Rapid filament prototyping of a bionic hand, IOP Conf. Series: Materials Science and Engineering 568 (2019)

4. https://en.wikipedia.org/wiki/Stereolithography

5. https://www.anycubic.com/pages/about-anycubic

6. X. Wang, M. Jiang, ZW. Zhou, JH. Gou and D. Hui - 3D printing of polymer matrix composites: A review and prospective, COMPOSITES PART B-ENGINEERING, Volume: 110, 442-458 (2017)

7. T. Babanatsas, R. M. Babanatis Merce, D. O. Glăvan and A. Glăvan - Contributions to optimization of storage and transporting industrial goods, IOP Conf. Series: Materials Science and Engineering 294 (2017),

8. D. O. Glavan , T. Babanatsas, M. Borzan, I. Radu and R. M. Babanatis Merce Considerations about command system for lathes with numerical controls, adaptive controls and copying system with hydraulic modules or computer assisted, IOP Conf. Series: Materials Science and Engineering 393 (2018),

9. X. Peng, L. Chaofeng, C. Xinwei and Z. Yanwu - UV-curring behavior of unsaturated cyclohexanone formaldehyde resins with thiols, Journal of Applied Polymer Science 137(9) : 48420 (2019)

10. A.Rami, Y. Hyung-In, L. Jason,... J. L. Sang - Shear bond strength of provisional repair materials bonded to 3d printed resin, Journal of Dental Sciences 16(1) (2020) 